\title{
Towards an Integrative "Lightness" of Being
}

\begin{abstract}
Editor's Note: With this column, JACM moves into the $5^{\text {th }}$ year of the Minding the Mind-body Literature column partnership with the Osher Center for Integrative Medicine at Harvard Medical School and Brigham and Women's Hospital. In e-convening with the author team on this column's focus - Center Executive Director Peter Wayne, PhD, Clinical Research Director Gloria Yeh, MD, MPH and Education Director Darshan Mehta, MD, MPH - an idea was quickly endorsed to, as the jazzman Mose Allison sang, "put the accent on the affirmative." The authors chose articles that examine recent research on such interventions as upping optimism, using laughter, and shifting imagery and intention. Have we been missing an (up)beat here?
\end{abstract}

- John Weeks, Contributing Editor, Special Projects and Collaborations, JACM (johnweeks-integrator.com).

\section{Lighten Up! How Imagery and Postural Intention Affect Balance and Muscle Tone in Older Adults}

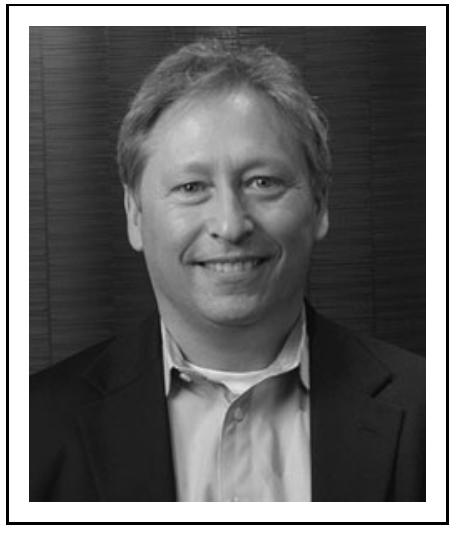

\section{Peter Wayne, PhD}

Director

Osher Center for Integrative Medicine

Harvard Medical School and Brigham and Women's Hospital

\footnotetext{
Central to MIND-BODY practices like tai chi, yoga, and Alexander technique are instructions that both inform postural alignments and biomechanics (e.g., vertical spine), but also the internal qualities that generate and result from them. For example, the tai chi classics, which espouse a lighter, less-can-be-more strategy to exercise, include statements such as, "the head should feel as if suspended from above, and the entire body will be light and agile." While a substantial body of research supports that cognitive processes such as imagery, visualization, and intention can have therapeutic impacts on objectively measured physiological and clinical outcomes, few studies have attempted to experimentally evaluate the impact of postural modifications
}

communicated through an instructor's imagery-based verbal instruction (separate from physical or visual instruction/ feedback), and whether the content of verbal instructions impact balance and postural control.

Cohen and colleagues conducted an elegant study that assessed the effects of brief postural instructions on static and dynamic balance in healthy older adults. ${ }^{1}$ Nineteen healthy adults over age 60 (mean age 69 years; 12 women, 7 men) were recruited and evaluated using a repeatedmeasures design to investigate how balance would be affected by instructing them to stand during each of three qualitatively different states. Using standardized scripts, the "Light" condition provided an invitation to allow the head 
to balance lightly on the top of the spine and prevent any downward pulling. It included phrases like 'let your head float at the top of your spine', and 'you can also expand in width'. The "Effortful" condition encouraged participants to use muscular effort and core strength to pull their heads up to their full height and their shoulders back. It referenced a strong military posture. The "Relax" condition guided a minimization of effort with no specific intention of postural uprightness. Instructions include 'stand as if you were feeling tired and lazy', and 'nobody is watching' and 'let everything settle downward'.

Impact of standing conditions were evaluated during tests of static and dynamic balance. Static balance examined sway (amplitude, frequency, path length, and jerkiness of the center of mass (COM), using an inertial sensor placed in the lumbar region while participants stood on a springy foam mat for 30 seconds. Dynamic balance examined kinematics and muscle activity during an in-place foot lift (raise the left foot off the floor, hold for $3 \mathrm{~s}$, release). During the dynamic task, motion capture cameras estimated standard parameters of COM dynamics. Surface electrodes also measured muscle activity on the standing side of the body.

Results supported robust effects of instructed states especially during the dynamics balance task. With respect to balance assessed by foot-in-air duration, 'Light' instructions led to the longest time while 'Effortful' exhibited the shortest. With respect to sway, 'Light' instructions exhibited $27 \%$ lower anteroposterior variability of the COM, compared to 'Effortful'. With respect to posture, 'Relax' instructions led to the farthest forward head position. Not surprisingly, torso muscle activity on the stance side of the body was higher in the 'Effortful' condition than in the other two conditions. During quiet stance testing, 'Effortful' instructions increased mediolateral jerk and path length.
Improving balance and preventing falls in older adults is a major public health priority for our aging boomer population. Mind-body techniques such as a tai chi, yoga, Alexander technique, Feldenkrais, among others, show promise (with various degrees of clinical evidence) in improving mobility and balance, and also in addressing musculoskeletal pain. However, the multimodal nature of these interventions poses challenges for understanding the relevance of specific intervention components (e.g., aerobic and muscle conditioning, breathing, mental focus, body positioning, postural qualities, instructor presence and language) on therapeutic outcomes. Extending prior research conducted in individuals with Parkinson's disease ${ }^{2}$ findings from this study support that verbal instructions, including imagery and visualization, can impact postural control and may be contribute the therapeutic value of mind-body exercises. These findings suggest that imageryand visualization-based instruction could be used to enhance the impact of conventional rehabilitation interventions and are effectively therapeutic modalities. They also highlight the need for consistent teaching protocols within mind-body studies, including careful attention to both what is said, as well as what is done. More broadly, these findings support an embodied cognition framework that appreciates rich interdependence of mental and physical processes.

Citations: 1. Cohen RG, Baer JL, Ravichandra R, Kral D, McGowan C, Cacciatore TW. Lighten Up! Postural Instructions Affect Static and Dynamic Balance in Healthy Older Adults. Innov Aging. 2020;24;4:igz056. DOI: 10.1093/ geroni/igz056.

2. Cohen RG, Gurfinkel VS, Kwak E, Warden AC, Horak FB. Lighten Up: Specific Postural Instructions Affect Axial Rigidity and Step Initiation in Patients With Parkinson's Disease. Neurorehabil Neural Repair. 2015;29:878-888. DOI: $10.1177 / 1545968315570323$.

\title{
Optimizing Optimism for Cardiovascular Health
}

\author{
Gloria Yeh, MD, MPH \\ Director of Clinical Research \\ Osher Center for Integrative Medicine \\ Harvard Medical School and Brigham and Women's Hospital \\ Director, Research Fellowship in Integrative Medicine \\ Division of General Medicine, Beth Israel Deaconess Medical Center
}

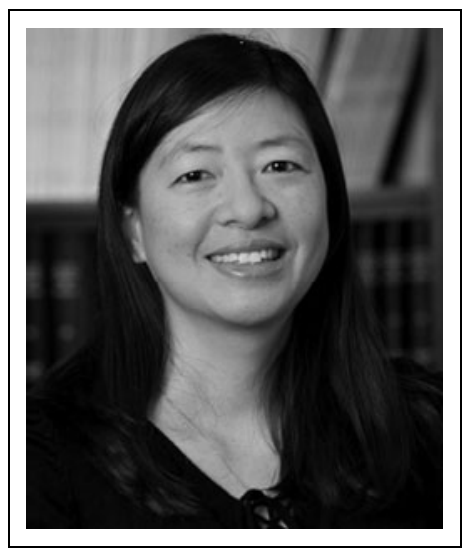

O PTIMISM IS COMMONLY defined as the tendency to believe that good things will happen in the future, and that bad events are less likely. Fascinating empirical studies suggest that optimistic individuals may respond to difficulty and adversity in more adaptive ways with conferred benefits in multiple life arenas (e.g., work, school, relationships). Positive associations have also been reported between optimism and various health outcomes including improved 
anxiety, pain, post-surgical complications, immune function, and even cardiovascular risk. Optimism is often described as a trait and can be stable across the lifespan starting in young adulthood. Importantly, optimism can be modifiable, and thus, it is a potential target for intervention.

Mind-body medicine acknowledges a natural connection between positive psychological states and overall health, however, it still tends to focus on pathology (e.g., stress, depression, anxiety) and the impact of negative mental states on health. This parallels medicine as a whole. Less attention is paid to positive states of well-being. While a positive mindset is central to many mind-body traditions, until recently, measures of optimism, kindness, prosociality, and flourishing were not typically included in research studies. There is now increasing recognition that well-being is not simply the absence of negative psychology or emotions and instead, positive factors or mindsets related to human flourishing- happiness, meaning, optimism- are equally important to consider. With these threads to positive psychology, the medical community is acknowledging the value of positive cognitive-emotional factors, and that these factors might confer independent therapeutic benefit.

The field of cardiology is a good example of this trend. Historically, studies have focused on the cardiovascular effects of negative psychological emotions. There is established evidence for distress-related morbidity and a robust literature on depression, anxiety, and social isolation, and their detrimental impact on cardiovascular risk. The assessment of positive psychological factors in cardiovascular disease is uncommon. Kubzansky and colleagues, however, have been exploring this line of inquiry for many years. Almost two decades ago they were the first to report an association between high optimism and lower cardiovascular risk, including angina, myocardial infarction, and even cardiovascular mortality- independent of depression or other psychological distress. ${ }^{1}$ Subsequent studies, mostly cross-sectional, have since continued to support the protective effects of optimism against incident cardiovascular disease and stroke.

In the current highlighted study, Kubzansky and colleagues provide further support for optimism as an important and impactful psychological factor for cardiovascular health. ${ }^{2}$ Investigators analyzed prospective data from a very large cohort of hypertension-free active-duty U.S. Army soldiers $(\mathrm{N}=103,486)$. The sample was relatively young (mean age $\sim 29$ years) and racially diverse (38\% nonwhite). Participants completed the Global Assessment Tool for psychological functioning (including a multi-item measure of optimism) annually and were followed up to five years for the development of hypertension. Separate nested models were developed, controlling for age, multiple potential confounders (e.g., diabetes, family history), and other variables along the pathway to cardiovascular disease (e.g., smoking, body-mass-index). Models were further adjusted for confounding by depression, which has been linked to both lower optimism and higher hypertension risk. Across the series of adjusted analyses, investigators found an 8-13\% decreased risk of incident hypertension with each one standard deviation increase in the optimism score. By optimism quintiles, there was a $34 \%$ reduction in hypertension risk for the highest vs. lowest quintiles with a dose-response relationship. The effect was largely similar across racial/ ethnic groups (including Black and Hispanic individuals) and independent of conventional cardiovascular risk factors, health behaviors, depression, length of deployment, and Army rank. Investigators propose optimism as a reasonable target for primordial prevention of cardiovascular disease.

The Austrian neurologist, psychiatrist and Holocaust survivor Viktor Frankl wrote of "tragic optimism" -optimism that allows one to find meaning and happiness even in the face of the deepest adversity (as many front-line soldiers have experienced), turning the trials of the human condition into human achievement. At the core of many mind-body contemplative traditions is a fundamental acknowledgement of the human condition. Critics have characterized optimists as being merely naïve or in denial of life's challenges. However, optimism may be a vehicle for deeper understanding, and congruent with the principles of mindful awareness, allow recognition of the positive in a healthy, adaptive way. Rather than a shallow view of optimism with blindly positive affirmations, "engineering optimism" through mind-body approaches offers another perspective. For patients with or at risk for cardiovascular disease, perhaps the lifestyle prescription should include a mindful cultivation of positive psychology, optimism, finding meaning, and hope. Considering that every $10 \mathrm{mmHg}$ reduction in systolic blood pressure leads to approximately $25 \%$ reduction in cardiovascular/stroke risk, a little dose of optimism could go a long way.

Medicine's increasing recognition of the value of positive health states is encouraging. Two programs with the Harvard Medical School/Harvard University network and part of the Osher Center's Without Walls community- the Human Flourishing Program and the Lee Kum Sheung Center for Health and Happiness (which Kubzansky co-directs)- provide examples of remarkable research and educational initiatives in this space. With these evolving trends towards positivity, one can't help but to feel optimistic about the future of medicine and the promise of mind-body approaches.

Citations: 1. Kubzansky LD, Sparrow D, Vokonas P, Kawachi I. Is the glass half empty or half full? A prospective study of optimism and coronary heart disease in the normative aging study. Psychosom Med Nov-Dec 2001;63:910-916. DOI: 10.1097/00006842-200111000-00009.

2. Kubzansky LD, Boehm JK, Allen AR, Vie LL, Ho TE, Trudel-Fitzgerald C, Koga HK, Scheier LM, Seligman MEP. Optimism and risk of incident hypertension: a target for primordial prevention. Epidemiol Psychiatr Sci. 2020;29:e157. DOI: 10.1017/S2045796020000621. 


\title{
"Can Laughter be Medicine?"
}

\author{
Darshan Mehta, MD, MPH \\ Director of Education \\ Osher Center for Integrative Medicine \\ Harvard Medical School and Brigham and Women's Hospital \\ Medical Director, Benson-Henry Institute for Mind Body Medicine \\ Massachusetts General Hospital
}

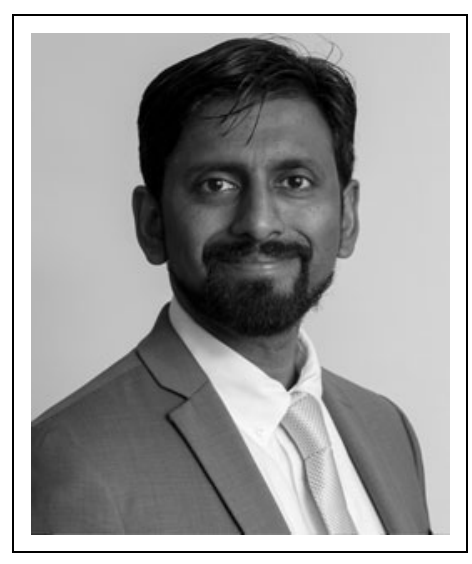

$\mathbf{T}$ HE HEALING POWER of laughter has been something of a mystery to scientists. In 1976, the journalist and UCLA faculty member, Norman Cousins came down with a painful degenerative disease that was initially diagnosed as ankylosing spondylitis. As his condition worsened, he checked into a hotel and treated himself with a regimen of intravenous vitamin $\mathrm{C}$, Marx Brothers films, The Three Stooges, and episodes of Candid Camera. Ten minutes of belly laughs calmed his pain for two hours; within weeks, his disease mysteriously faded into remission. This healing power of laughter was famously detailed in his groundbreaking book, Anatomy of an Illness. Studies have found that children laugh 300 times per day (peaking at age 5), whereas adults laugh only 20 times per day.

If laughter is healing, we also know that the converse is true. Individuals with chronic medical conditions suffer from much higher rates of depression. And individuals with untreated or poorly treated depression have much greater challenges in the management of their chronic medical condition. This has been true in the management of chronic kidney disease, including individuals requiring hemodialysis. With that being said, hemodialysis patients are a captive audience. And so, when they are sitting in a chair for 3-4 hours, this presents a unique opportunity to intervene.

In this prospective randomized control study by Bennett et al., 151 participants from 10 hemodialysis centers were randomized to hemodialysis with laughter therapy, or to usual care. Given that the intervention was performed on a group-basis, randomization was performed at the level of the center, rather than at the individual. Laughter therapy consisted of 8 weekly sessions; they began approximately 1 hour after the start of their hemodialysis treatment. Each 30-minute session consisted of: breathing and stretching exercises, along with facilitated laughter exercises; the intervention was based upon a prior published study. The weekly sessions were led by two certified laughter yoga leaders. What is particularly striking about this pragmatic study is that the hemodialysis staff were also educated in the protocol, and invited to take part as well. Moreover, this study included patients whose first language was not English - an understated but notable strength of this study.

The study investigators used the Patient Health Questionnaire (PHQ-4) to assess change immediately before the first laughter therapy session and following the final session. The primary outcome was the depression symptom subscale of the PHQ-4. Secondary outcomes included the anxiety subscale of the PHQ-4, subjective well-being (as measured by the personal wellbeing index), and the London evaluation of illness (LEVIL) instrument. Of note, the study obtained a waiver of consent through their independent review board. This was obtained by the authors' prior studies establishing the low-risk nature of the intervention, as well as the fact the consent process would bias participation (i.e., explanation of the benefits of laughter itself). However, in keeping with the ethics review, no individual-level demographic data was analyzed.

The proportion of patients who reported depressive symptoms decreased from $22 \%$ to $20 \%$ in the control group and $17 \%$ to $8 \%$ in the laughter intervention group. The change in proportion was statistically significant $(\mathrm{p}=0.04)$. There was no statistically significant treatment effect found in the other patient reported outcome measure scores (anxiety, subjective well-being, and the LEVIL instrument).

This study is an important contribution for several reasons. First, in a condition such as chronic kidney disease, where depression is a major comorbidity, this study provides important pilot data for future work. Second, this study is truly pragmatic. There is no practical way of "blinding" someone from laughter, and underscores challenges in research design. Finally, given the completely benign side effect profile, along with ease of delivery, there is broad application and generalizability of the results. We do not know how different the treatment and control groups may have been, as the investigators did not have that data; this is an acknowledged limitation of the study.

Patch Adams's work and his biopic raised the public awareness of how laughter can be "medicalized." And future studies are needed to further understand the clinical benefits of laughter therapy. Laughter is a universally human experience; it cuts across age, race, ethnicity, gender, and language. In that sense, this study modeled inclusivity. Moreover, there is an "infectious" nature to laugh. As such, the group effect cannot be ignored; and, if anything, it is vital to the success of the intervention. Finally, this study included staff involvement - in thinking about healthcare worker well-being, perhaps there may be another story to be told here, as we are left wondering how they also changed through the intervention.

Citation: 1. Bennett PN, Hussein WF, Reiterman M, Yu J, Schiller B. The effects of laughter therapy on depression symptoms in patients undergoing center hemodialysis: A pragmatic randomized controlled trial. Hemodial Int. 2020;24:541-549. 